\title{
Estudio teórico-experimental de la dinámica del movimiento circular con fricción
}

\author{
R. Cortés-Maldonado ${ }^{a, *}$, M. C. Gómez-Conde ${ }^{b}$, C. Bueno-Avendaño ${ }^{a}$, y J. F. Casco-Vásquez ${ }^{a}$ \\ ${ }^{a}$ Tecnológico Nacional de Méxicol IT de Apizaco, \\ Ave. Instituto Tecnológico s/n, Apizaco, 90300 Tlaxcala, México, \\ *e-mail:raul.cm@apizaco.tecnm.mx \\ ${ }^{b}$ Universidad Politécnica de Puebla, Maestría en Enseñanza de las Ciencias, \\ Tercer carril del ejido "Serrano"s/n, San Mateo Cuanalá. Juan C. Bonilla, 72640. Puebla-México.
}

Received 20 February 2021; accepted 19 April 2021

\begin{abstract}
En este trabajo se presenta un estudio teórico-experimental de la dinámica del movimiento de una esfera que se mueve en una trayectoria circular vertical con fricción. En el estudio teórico se resuelve una ecuación diferencial de Bernoulli que permite calcular la rapidez en cualquier posición angular y la rapidez mínima necesaria para que la esfera complete una vuelta en la trayectoria circular. En el estudio experimental se utilizan fotopuertas para medir la posición angular de la esfera como función del tiempo; a partir de esto, se obtienen de manera indirecta la rapidez y fuerza normal en cualquier posición angular. Al comparar los resultados teóricos y experimentales obtenidos de forma indirecta para la rapidez y fuerza normal se observa que el modelo teórico describe de forma cualitativa el comportamiento experimental cuando $\mu=0.12$. Finalmente, se analiza el comportamiento teórico de la rapidez y fuerza normal para distintos valores del coeficiente de fricción cinética, donde se observa que para $\mu \geq 0.2$ la rapidez y fuerza normal final decaen de manera abrupta.
\end{abstract}

Descriptores: Rapidez; fuerza normal; fricción; ecuación de Bernoulli.

This work presents a theoretical and experimental study of the dynamics of the motion of a sphere that moves in a vertical circular trajectory with friction. In the theoretical study, a Bernoulli differential equation is solved to calculate the velocity at any angular position and the minimum velocity necessary for the sphere to complete one turn in the circular track. In the experimental study, photogates are used to measure the angular position of the sphere as a function of time, from which the velocity and normal force are indirectly obtained in any angular position. When comparing the theoretical and experimental results obtained indirectly for the velocity and the normal force, it is observed that the theoretical model qualitatively describes the experimental behavior when $\mu=0.12$. Finally, the theoretical behavior of the speed and normal force is analyzed for different values of the coefficient of friction kinetic, where it is observed that for $\mu \geq 0.2$ the final normal speed and force decline abruptly.

Keywords: Velocity; normal force; friction; Bernoulli equation.

DOI: https://doi.org/10.31349/RevMexFisE.18.020204

\section{Introducción}

Un problema clásico en cursos de mecánica básica de nivel superior es aquel donde se requiere calcular la rapidez mínima para que una partícula complete una vuelta en un aro vertical. Este ejercicio se resuelve de manera sencilla al despreciar la fricción, aplicar la segunda ley de Newton y mediante la conservación de la energía mecánica para obtener $\sqrt{5 R g}$, donde $g$ es la aceleración debido a la gravedad [1,2]. Este problema ha sido ampliamente estudiado en la literatura y se han propuesto soluciones alternativas a partir de consideraciones geométricas, utilizando los conceptos de serie de Taylor y radio de curvatura, así como las nociones de límite de una función continua y funciones trigonométricas, además de elementos de geometría euclidiana y la congruencia de ángulos en triángulos isósceles [3,4].

La complejidad se incrementa cuando se considera la presencia de fricción, por ello, este problema y sus variantes pocas veces se abordan en cursos de licenciatura, sin embargo, dicho problema también ha sido ampliamente estudiado en la literatura [5-8]. A pesar de esto, el tema sigue siendo de interés desde el punto de vista teórico, experimental y educativo [9-13].

En este trabajo se presenta un estudio teóricoexperimental de la dinámica del movimiento circular con fricción. La Sec. 2 presenta el marco teórico para resolver de manera exacta el problema de calcular la rapidez mínima de entrada para que una partícula complete una vuelta en una trayectoria circular vertical cuando el coeficiente de fricción cinética es a) cero y b) distinto de cero. En este último caso, el análisis da como resultado una ecuación diferencial de Bernoulli que, al resolverla, permite calcular la rapidez en cualquier posición angular. La Sec. 3 describe una configuración experimental simple que permite medir la posición angular de la esfera como función del tiempo y a partir de este resultado se calculan la rapidez y fuerza normal para cualquier posición angular. Posteriormente, se comparan los resultados experimentales y teóricos para la rapidez y fuerza normal a lo largo de la trayectoria. También se presentan las predicciones teóricas del comportamiento de la rapidez y fuerza normal para distintos valores del coeficiente de fricción. Finalmente, en la Sec. 4 se presentan las conclusiones del trabajo. 


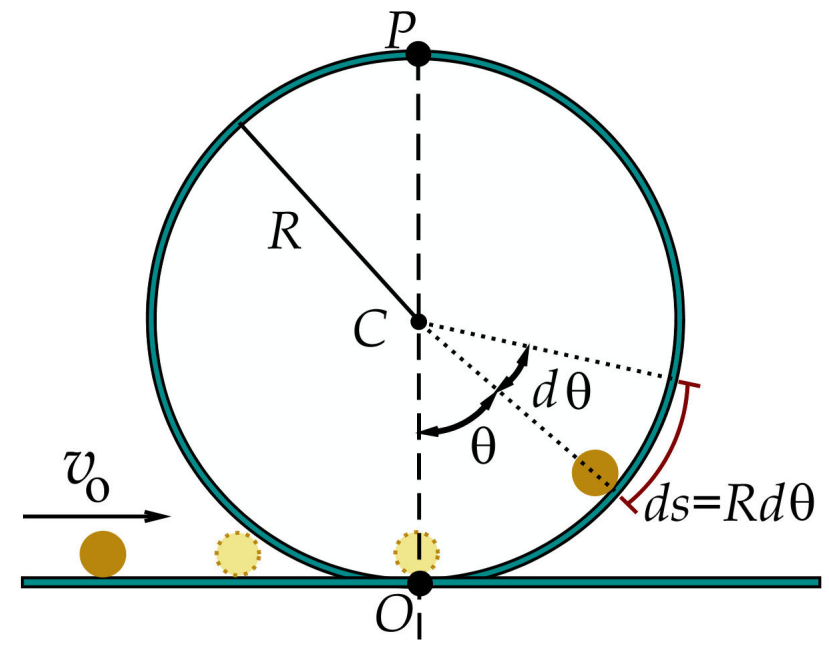

FIGURA 1. Una esfera de masa $m$ se mueve con rapidez inicial $v_{o}$ en un aro vertical de radio $R$.

\section{Marco teórico}

Se considera un aro vertical de radio $R$ (ver Fig. 1) descrito por las ecuacioens paramétricas

$$
\begin{aligned}
& x=R \sin \theta \\
& y=R(1-\cos \theta) .
\end{aligned}
$$

Se requiere determinar la rapidez mínima $v_{o}$ con la que una esfera de masa $m$ debe lanzarse para dar una vuelta completa en el aro vertical.

La Fig. 2 muestra el diagrama de cuerpo libre para la esfera en una posición arbitraria $\theta$. Las fuerzas que actúan son el peso, $W=m g$, la fuerza normal $F_{N}$ y la fuerza de fricción modelada como $f=\mu F_{N}$, donde $\mu$ es el coeficiente de fricción cinética.

De acuerdo con la segunda ley de Newton expresada en coordenadas tangencial y normal, se obtiene

$$
\begin{aligned}
& \Sigma F_{t}=-m g \sin \theta-\mu F_{N}=m v \frac{d v}{d s}, \\
& \Sigma F_{n}=F_{N}-m g \cos \theta=\frac{m v^{2}}{R},
\end{aligned}
$$

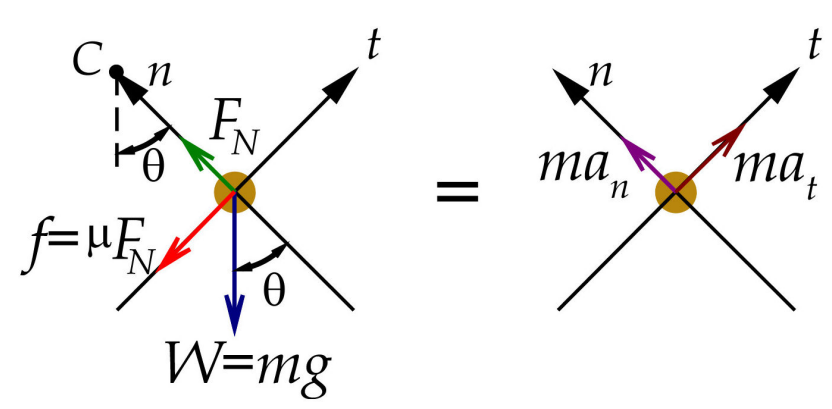

FIGURE 2. Segunda ley de Newton en coordenadas tangencial y normal para una esfera de masa $m$ en una posición arbitraria $\theta$. donde $v$ es la rapidez y depende de la posición angular $\theta$, esto es $v=v(\theta)$, y $d s$ es un elemento diferencial de longitud de arco para una circunferencia, dado por $d s=R d \theta$.

\subsection{Solución exacta sin fricción, $\mu=0$}

En primer lugar se analiza el caso sin fricción, que corresponde a $\mu=0$. Además, a partir de las Ecs. (2-3) y de la expresión $d s=R d \theta$, se obtiene la siguiente ecuación diferencial oridinaria:

$$
-m g \sin \theta=m v \frac{1}{R} \frac{d v}{d \theta} .
$$

A continuación, al separar variables e integrar desde una rapidez inicial $v_{o}$ hasta una rapidez final $v$ y desde una posición inicial $\theta_{o}=0$ hasta una posición final $\theta$, se obtiene

$$
\begin{gathered}
\int_{v_{o}}^{v} v^{\prime} d v^{\prime}=-R g \int_{0}^{\theta} \sin \theta^{\prime} d \theta^{\prime} \\
\frac{1}{2}\left(v^{2}-v_{o}^{2}\right)=-R g(-\cos \theta+1),
\end{gathered}
$$

o bien,

$$
v^{2}=v_{o}^{2}-2 R g(1-\cos \theta) .
$$

Ahora, al sustituir la Ec. (6) en (3) y despejando a $F_{N}$ se obtiene

$$
F_{N}=m g \cos \theta+\frac{m}{R}\left(v_{o}^{2}-2 R g(1-\cos \theta)\right) .
$$

La esfera permanecerá en el aro vertical siempre y cuando la fuerza normal $F_{N}$ sea distinta de cero; en caso contrario, si la fuerza normal se hace cero; la esfera perderá el contacto con la trayectoria circular y caerá. Al suponer que este último caso ocurre en el punto $P$, por consiguiente $F_{N P}=0$, la coordenada angular es $\theta_{P}=\pi$ rad y la rapidez inicial para alcanzar el punto $P$ será $v_{o}=v_{P}$. Al sustituir estos valores en la Ec. (6) se obtiene

$$
m g=\frac{m}{R}\left(v_{P}^{2}+4 R g\right),
$$

y entonces se verifica fácilmente que $v_{P}$ es

$$
v_{P}=\sqrt{5 R g} .
$$

Por lo tanto, la rapidez de entrada $v_{o}$ para que la esfera complete una vuelta en el aro vertical debe ser mayor que $v_{P}$, esto es $v_{o}>v_{P}$. Este resultado es independiente de la masa de la esfera y puede obtenerse también de manera muy sencilla aplicando la conservación de la energía mecánica. 


\subsection{Solución exacta con fricción $\mu \neq 0$}

La situación general que considera la presencia de fricción entre la esfera y el aro se describe en las Ecs. (2-3). A partir de estas ecuaciones y de la expresión para un elemento diferencial de longitud de arco, $d s=R d \theta$, se obtiene la siguiente ecuación diferencial:

$$
-m g \sin \theta-\mu\left(m g \cos \theta+\frac{m v^{2}}{R}\right)=m v \frac{1}{R} \frac{d v}{d \theta} .
$$

Es importante comparar las Ec. (4) y (10); nótese cómo se incrementa notablemente la complejidad de la ecuación diferencial a resolver. En particular, la Ec. (10) se puede escribir como

$$
\frac{d v}{d \theta}=-\mu v-R g(\sin \theta+\mu \cos \theta) v^{-1},
$$

la cual tiene la forma de una ecuación de Bernoulli ${ }^{i}$ con $n=-1$ y $v=v(\theta)$.

Al definir $z=v^{2}$, multiplicar la Ec. (11) por $2 v$ y reordenar, se obtiene

$$
\frac{d z}{d \theta}+2 \mu z=-R g(\sin \theta+\mu \cos \theta) .
$$

La Ec. (12) es una ecuación diferencial lineal ordinaria que se resuelve al multiplicar ambos lados de la ecuación por el factor integrante $e^{2 \mu \theta}$, a partir de lo cual se obtiene

$$
\frac{d}{d \theta}\left(e^{2 \mu \theta} z\right)=-2 R g e^{2 \mu \theta}(\sin \theta+\mu \cos \theta) .
$$

A continuación se separan variables y se integra respecto a $\theta$, así el resultado es

$$
\begin{aligned}
e^{2 \mu \theta} z & =-\frac{2 R g e^{2 \mu \theta}}{4 \mu^{2}+1} \\
& \times\left[\left(2 \mu^{2}-1\right) \cos \theta+3 \mu \sin \theta\right]+C,
\end{aligned}
$$

donde $C$ es una constante de integración que se calcula a partir de la condición inicial $\left.z\right|_{\theta=0}=v_{o}^{2}$ y tiene el valor

$$
C=v_{o}^{2}+\frac{2 R g\left(2 \mu^{2}-1\right)}{4 \mu^{2}+1} .
$$

$\mathrm{Al}$ sustituir $C$ en la Ec. (14) y despejar a $z=v^{2}$ se obtiene

$$
z=v^{2}=e^{-2 \mu \theta}\left[v_{o}^{2}+\frac{2 R g\left(2 \mu^{2}-1\right)}{4 \mu^{2}+1}\right]-\frac{2 R g}{4 \mu^{2}+1}\left[\left(2 \mu^{2}-1\right) \cos \theta+3 \mu \sin \theta\right]
$$

O bien,

$$
v=\sqrt{e^{-2 \mu \theta}\left[v_{o}^{2}+\frac{2 R g\left(2 \mu^{2}-1\right)}{4 \mu^{2}+1}\right]-\frac{2 R g}{4 \mu^{2}+1}\left[\left(2 \mu^{2}-1\right) \cos \theta+3 \mu \sin \theta\right]}
$$

Al sustituir la Ec. (16) en (3) se obtiene,

$$
F_{N}=m g \cos \theta+\frac{m}{R}\left[e^{-2 \mu \theta}\left[v_{o}^{2}+\frac{2 R g\left(2 \mu^{2}-1\right)}{4 \mu^{2}+1}\right]-\frac{2 R g}{4 \mu^{2}+1}\left[\left(2 \mu^{2}-1\right) \cos \theta+3 \mu \sin \theta\right]\right] .
$$

Al igual que en el caso con $\mu=0$, la esfera permanecerá en contacto con el aro vertical siempre que $F_{N} \neq 0$. Al suponer que la esfera cae justo cuando llega al punto $P$, esto significa que $F_{N P}=0$, la coordenada angular es $\theta_{P}=\pi \operatorname{rad}$ y la rapidez inicial para alcanzar el punto $P$ será $v_{o}=v_{P}$. Al sustituir estos valores en la Ec. 18 se obtiene

$$
\begin{aligned}
m g & =\frac{m}{R}\left(e^{-2 \mu \pi}\right. \\
& \left.\times\left[v_{P}^{2}+\frac{2 R g\left(2 \mu^{2}-1\right)}{4 \mu^{2}+1}\right]+\frac{2 R g\left(2 \mu^{2}-1\right)}{4 \mu^{2}+1}\right) .
\end{aligned}
$$

Después de manipular matemáticamente la ecuación anterior se obtiene

$$
v_{P}=\sqrt{\frac{R g}{4 \mu^{2}+1}\left(-4 \mu^{2}+3 e^{2 \mu \pi}+2\right)} .
$$

Nótese que la rapidez $v_{P}$ sigue sin depender de la masa de la esfera, no obstante, depende del coeficiente de fricción y del radio del aro.

Por lo tanto, la rapidez de entrada $v_{o}$ para que la esfera complete una vuelta en el aro vertical debe ser mayor que $v_{P}$, esto es $v_{o}>v_{P}$.

Se puede verificar fácilmente que si $\mu=0$, la Ec. (18) se reduce a $v_{P}=\sqrt{5 R g}$, que es el resultado para el caso ideal cuando se desprecia la fricción.

\section{Experimento y comparación con resultados teóricos}

En la presente sección se compara la rapidez de la esfera obtenida a partir de la solución exacta del modelo dinámico del aro vertical con fricción (Ec. (17)) con la rapidez obtenida de forma indirecta a partir de mediciones de la posición angular de la esfera como función del tiempo. 


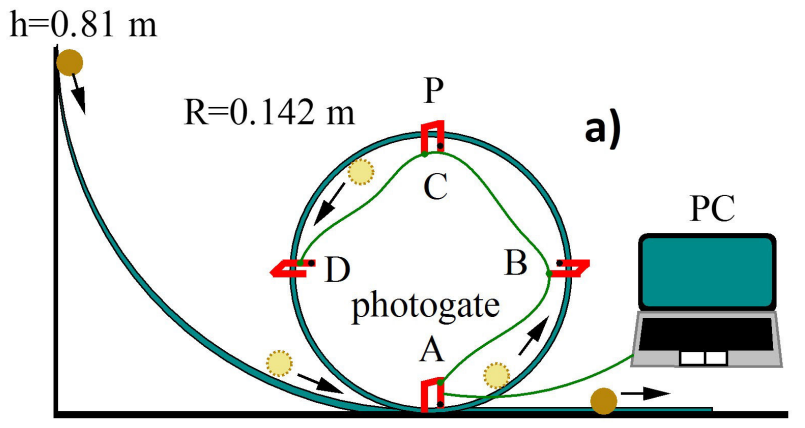

$\mathrm{O}$

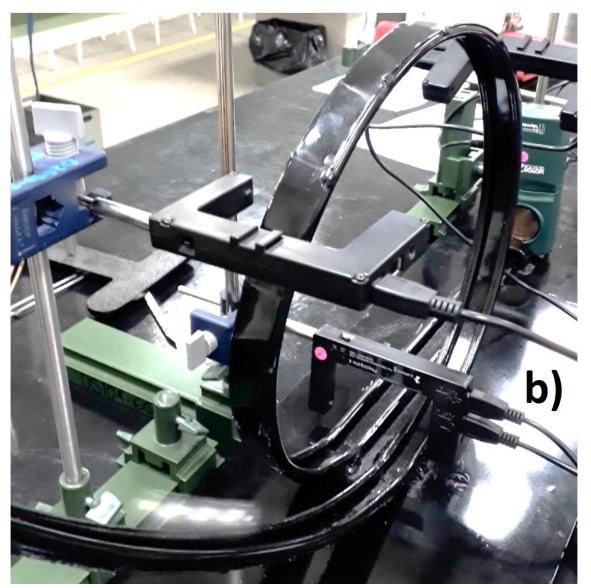

FIgURA 3. a) Configuración experimental general. b) Configuración experimental para medir el tiempo en las posiciones $\theta=$ $0^{\circ}, 120^{\circ}, 240^{\circ}$ y $360^{\circ}$, nótese que únicamente se utilizan tres fotopuertas para esta configuración.

\subsection{Experimento}

Las mediciones experimentales se realizaron con una esfera de masa $m=0.067 \mathrm{~kg}$ y diámetro $d=25.5 \mathrm{~mm}$. La esfera se suelta desde el reposo a una altura $h=0.81 \mathrm{~m}$ y pasa a través de un aro vertical de radio $R=0.142 \mathrm{~m}$.

La Fig. 3 muestra el montaje experimental que permite medir la posición de la esfera como función del tiempo. Para esto se han utilizado sensores infrarrojos (fotopuerta o photogate) de la marca Vernier y una PC con el software ScienceCube, disponibles en el laboratorio de Física del TecNM/I.T. de Apizaco. La fotopuerta produce una señal en estado alto cuando la luz infrarroja pasa libremente del emisor al receptor, mientras que produce una señal en estado bajo cuando se obstruye el paso. Para medir el tiempo durante el cual la esfera pasa por una posición específica, se considera el punto medio entre la señal de estado alto y la señal de estado bajo de la fotopuerta.

La fotopuerta A se conecta directamente a la PC, después a partir de A se conectan en serie las fotopuertas B, C y D. De acuerdo con el fabricante, el número máximo de fotopuertas en este modo es de 4.

La fotopuerta A se mantiene fija en $\theta=0^{\circ}$, posición que coincide $\operatorname{con} \theta=360^{\circ}$. Las fotopuertas B, C, y D se ajustan de modo que se puedan ubicar en distintas posiciones. Así,

\begin{tabular}{ccc}
\hline \hline TABLA I. Tiempo medido en cada posición anugular. \\
\hline $\begin{array}{c}\text { Posición } \\
\text { angular } \\
\text { (grados) }\end{array}$ & $\begin{array}{c}\text { Posición } \\
\text { angular } \\
(\mathrm{rad})\end{array}$ & $\begin{array}{c}\text { Tiempo } \\
\text { medido }\end{array}$ \\
\hline 0 & 0 & $(\mathrm{~s})$ \\
45 & $\pi / 4$ & 0.365 \\
90 & $\pi / 2$ & 0.388 \\
120 & $\pi / 3$ & 0.449 \\
135 & $3 \pi / 4$ & 0.466 \\
180 & $\pi$ & 0.478 \\
225 & $5 \pi / 4$ & 0.571 \\
240 & $4 \pi / 3$ & 0.599 \\
270 & $3 \pi / 2$ & 0.646 \\
315 & $7 \pi / 4$ & 0.656 \\
360 & $2 \pi$ & 0.685 \\
\hline
\end{tabular}

por ejemplo, en la configuración experimental de la Fig. 3a se puede medir el tiempo para las posiciones $\theta=0^{\circ}, 90^{\circ}$, $180^{\circ}, 225^{\circ}$ y $360^{\circ}$. En el caso del panel b) se puede medir el tiempo para las posiciones $\theta=0^{\circ}, 120^{\circ}, 240^{\circ}$ y $360^{\circ}$. En total se utilizaron cinco configuraciones experimentales distintas para las fotopuertas B, C y D para medir el tiempo en las posiciones $\theta=45^{\circ}, 90^{\circ}, 120^{\circ}, 135^{\circ}, 180^{\circ}, 225^{\circ}, 240^{\circ}, 270^{\circ}$ y $315^{\circ}$.

En cada configuración, el lanzamiento de la esfera se ha realizado 15 veces, siempre sincronizando el inicio de la medición en la PC con el lanzamiento manual de la esfera. Las mediciones se verificaron de modo que los tiempos medidos no cambiaran más de 0.02 segundos. Posteriormente, se promediaron los 15 valores de tiempo medidos en cada fotopuerta (posición) para obtener los valores motrados en la Tabla I.

La Fig. 4 muestra en puntos negros los datos experimentales, columnas dos y tres de la Tabla I, con sus respectivas

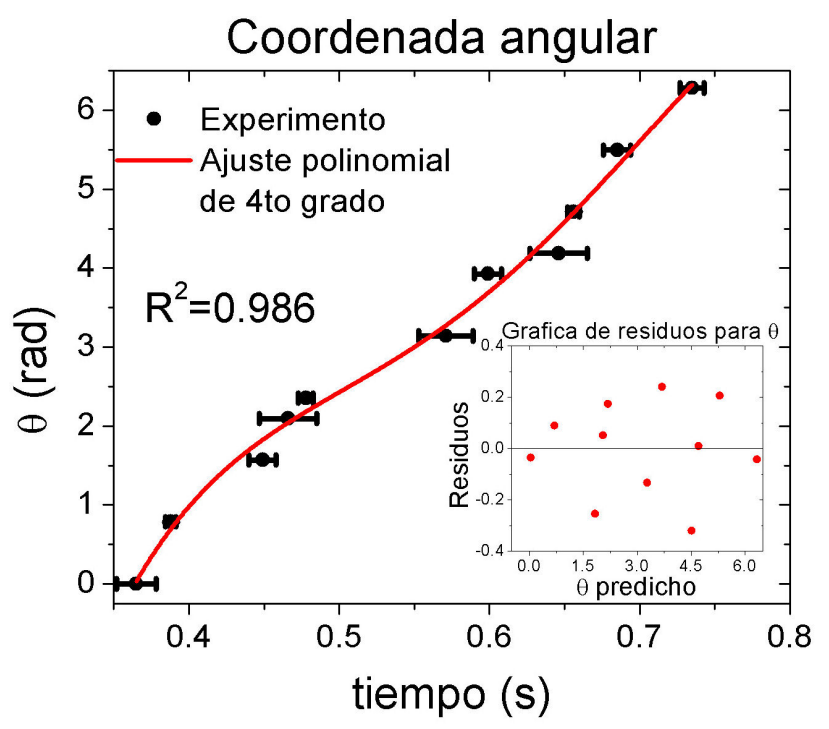

FIGURA 4. Experimento. 


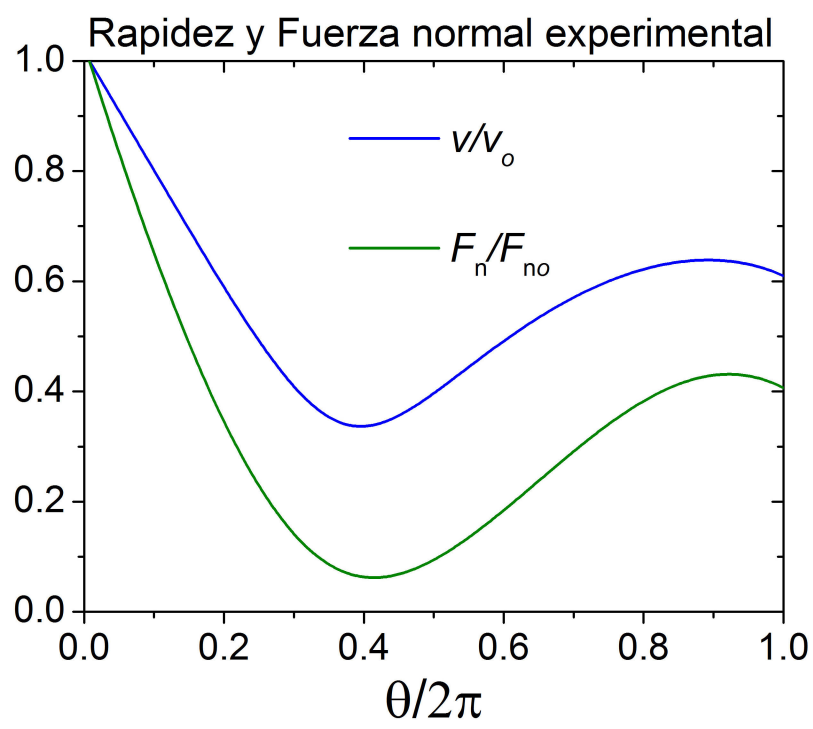

FIGURA 5. Rapidez y fuerza normal experimental.

barras de error, que no superan el valor de $\pm 0.02 \mathrm{~s}$ en el tiempo y \pm 0.05 rad en el ángulo. Para continuar el análisis es necesario realizar un ajuste que permita conocer la posición angular como función del tiempo, $\theta=\theta(t)$. Al considerar la presencia de la fricción, además de que el aro donde se realizaron las mediciones no es una circunferencia perfecta, sino una elipse con $e \approx 0$, se tiene que la dependencia angular de la rapidez exhibe al menos un punto de inflexión [11]. Por lo antes mencionado, se seleccionó un ajuste polinomial de cuarto grado con un coeficiente de determinación $R^{2}=0.986$ (curva en color rojo),

$$
\theta(t)=-654.4 t^{4}+1574 t^{3}-1382 t^{2}+539.7 t-77.75 .
$$

El recuadro de la Fig. 4 muestra la gráfica de residuos para $\theta$. Los residuos exhiben un comportamiento aleatorio, esto indica que el modelo es fiable.

La rapidez de la esfera en cualquier instante de tiempo se determina fácilmente como,

$$
v=R \frac{d \theta}{d t}=R\left(-2617.6 t^{3}+4722 t^{2}-2764 t+539.7\right),
$$

a esta ecuación la llamamos rapidez experimental. La fuerza normal experimental se obtiene de manera sencilla como

$$
F_{n}=m \frac{v^{2}}{R}=m \frac{\left(R \frac{d \theta}{d t}\right)^{2}}{R} .
$$

La Fig. 5 muestra las gráficas para la rapidez y fuerza normal obtenidas a partir de las Ecs. (22 23), en el intervalo de tiempo $[0.365,0.735]$ de segundos con pasos de 0.001. Ambas gráficas estań normalizadas con su correspondiente valor inicial.

La rapidez de la esfera en la entrada del aro vertical es $v_{o}$. Se observa que este valor decrece a medida que la esfera recorre la trayectoria circular en el intervalo de $(\theta / 2 \pi)=0$ hasta $(\theta / 2 \pi)=0.39$, en donde ocurre un mínimo local con el $33 \%$ de su valor inicial, poco antes de completar media vuelta. En el intervalo [0.39, 0.89] la rapidez exhibe un comportamiento creciente, hasta alcanzar el $63.8 \%$ de su valor inicial en $(\theta / 2 \pi)=0.89$. La rapidez vuelve a decrecer en el intervalo $[0.89,1]$; al finalizar la vuelta, la rapidez corresponde solo al $61 \%$ del valor inicial.

La fuerza normal en la entrada del aro vertical se denota por $F_{n o}$. En comparación con la rapidez, la fuerza normal exhibe un comportamiento decreciente más pronunciado en el intervalo de $(\theta / 2 \pi)=0$ hasta $(\theta / 2 \pi)=0.41$, en donde ocurre el mínimo local con el $6 \%$ del valor inicial. En el intervalo $[0.41,0.92]$ la fuerza normal crece hasta un máximo local ubicado en $(\theta / 2 \pi)=0.92$, después de este valor la fuerza normal decrece nuevamente hasta finalizar una vuelta con el $40 \%$ de su valor inicial.

\subsection{Predicciones teóricas}

La Fig. 6 panel a) muestra la rapidez de la esfera obtenida a partir de las Ecs. (17) y (22). La rapidez inicial para la Ec. (17) se ha ajustado a $v_{0}=1.1 v_{P}$ y el coeficiente de fric-
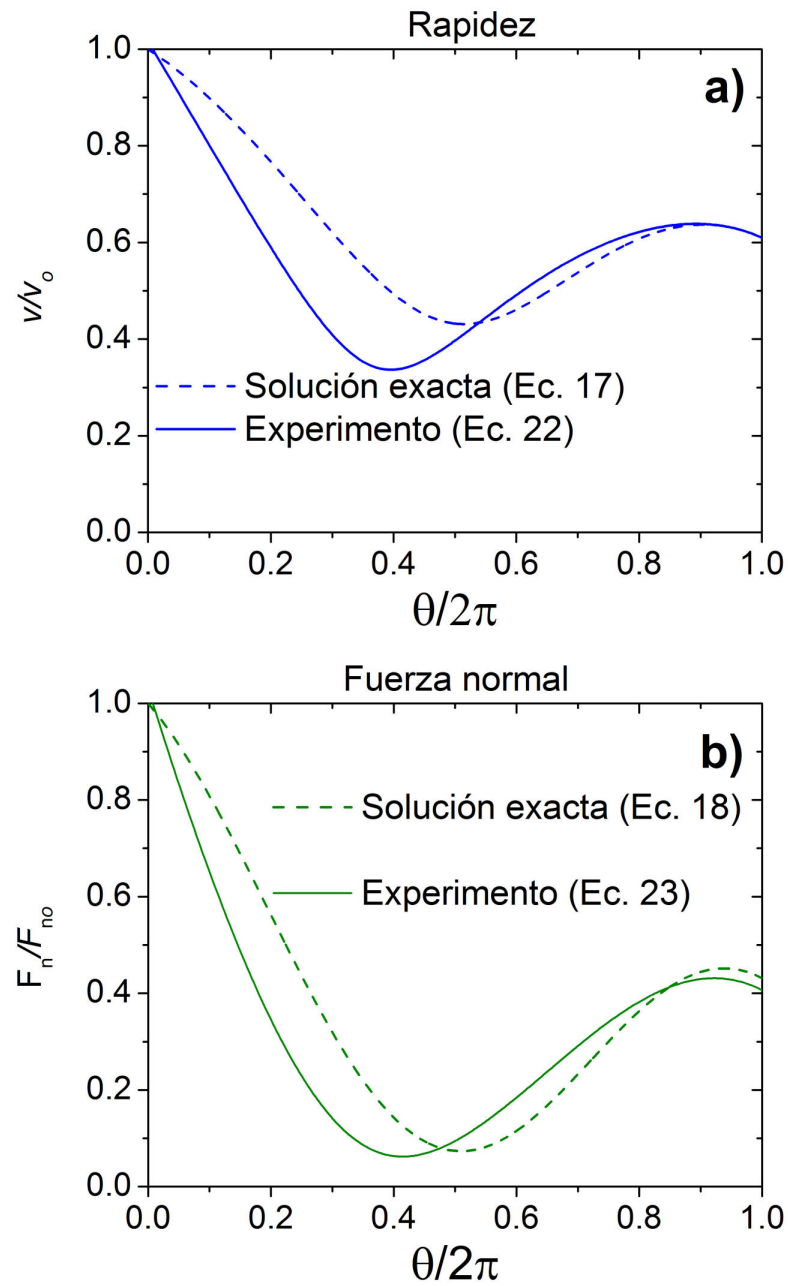

FIgURA 6. a) Rapidez exacta y experimental. b)Fuerza normal exacta y experimental. 
ción cinética a $\mu=0.12$, de manera que estos valores permiten reproducir cualitativamente los resultados experimentales. Se observa que el error entre la teoría y el experimento se incrementa de forma exponencial en el intervalo de $0<(\theta / 2 \pi)<0.3$, alcanzando el valor máximo de $21 \%$ en $(\theta / 2 \pi) \approx 0.3$. En el intervalo $0.3<(\theta / 2 \pi)<0.5$ el error decae notablemente, de tal modo que en $0.5<(\theta / 2 \pi) \leq 1$ el error es menor al $4 \%$. También se puede observar que existe una diferencia del $10 \%$ entre la teoría y el experimento para el valor mínimo de la rapidez y para la ubicación del mismo, $\left(v_{\exp } / v_{0, \exp }\right)=0.43,\left(v_{\text {teo }} / v_{0, \text { teo }}\right)=0.33 \mathrm{y}$ $\left(\theta_{\exp } / 2 \pi\right)=0.4,\left(\theta_{\text {teo }} / 2 \pi\right)=0.5$, respectivamente.

El panel b) de la Fig. 6 muestra la fuerza normal obtenida a partir de las Ecs. (18) y (23). Puede observarse que el error entre la teoría y el experimento también se incrementa exponencialmente en el intervalo de $0<(\theta / 2 \pi)<0.2$, el valor máximo es del $21 \%$ y ocurre en $(\theta / 2 \pi) \approx 0.2$. En el intervalo $0.2<(\theta / 2 \pi)<0.5$ el error disminuye de forma considerable y en $0.5<(\theta / 2 \pi) \approx \leq 1$ el error es menor al $7 \%$. En este mismo panel, se observa que el valor mínimo de la fuerza normal es del mismo orden de magnitud para el experimento y la teoría y que su ubicación también difiere en $10 \%$.

El origen del error observado en las gráficas teóricas y experimentales de la Fig. 6, se atribuye a que el aro donde se realizaron las mediciones no es una circunferencia perfecta, sino una elipse con $e \approx 0$. Se ha reportado que en trayectorias elípticas horizontales y verticales la rapidez presenta más de un punto de inflexión, de manera que el primero puede ocurrir en $(\theta / 2 \pi)<0.5$ [11].

Por otra parte, si considera al coeficiente de fricción como un parámetro independiente, cuyo valor numérico está determinado por los materiales involucrados en el sistema, resulta de interés analizar las predicciones teóricas del comportamiento de la rapidez y la fuerza normal obtenidas a partir de la Ec. (17).

La Fig. 7 muestra estas predicciones para valores propuestos de $\mu$ entre 0 y 1 en intervalos de 0.2 (ver paneles a y b). Para el caso sin fricción, $\mu=0$, la rapidez y fuerza normal tienen su mínimo justo en la mitad de la vuelta, además, su valor final coincide con el valor inicial, como es de esperarse. Para $\mu=0.2$ la rapidez y fuerza normal final disminuyen drásticamente hasta el $44 \%$ y $25 \%$ de su valor inicial, respectivamente. A medida que $\mu$ se incrementa, la rapidez y fuerza normal final continuan disminuyendo, de modo que para $\mu=0.4$, la rapidez final ha decaido hasta el $19 \%$ de su valor inicial y la fuerza normal hasta el $10 \%$. En ambos casos, el mínimo de la rapidez y fuerza normal ocurre en $(\theta / 2 \pi)=0.5$. En este punto se observa que a pesar de la disminución en la rapidez, la esfera no pierde contacto con la trayectoria circular. Además, se puede observar que el máximo presente en la segunda mitad de la vuelta disminuye en amplitud y se observa un corrimiento a valores menores de $(\theta / 2 \pi)$ a medida que el valor de $\mu$ crece. Para $\mu=0.6$, en el intervalo $0.4 \leq(\theta / 2 \pi) \leq 0.65$, la rapidez es tan pequeña que la esfera pierde contacto con la trayectoria, incorporándose
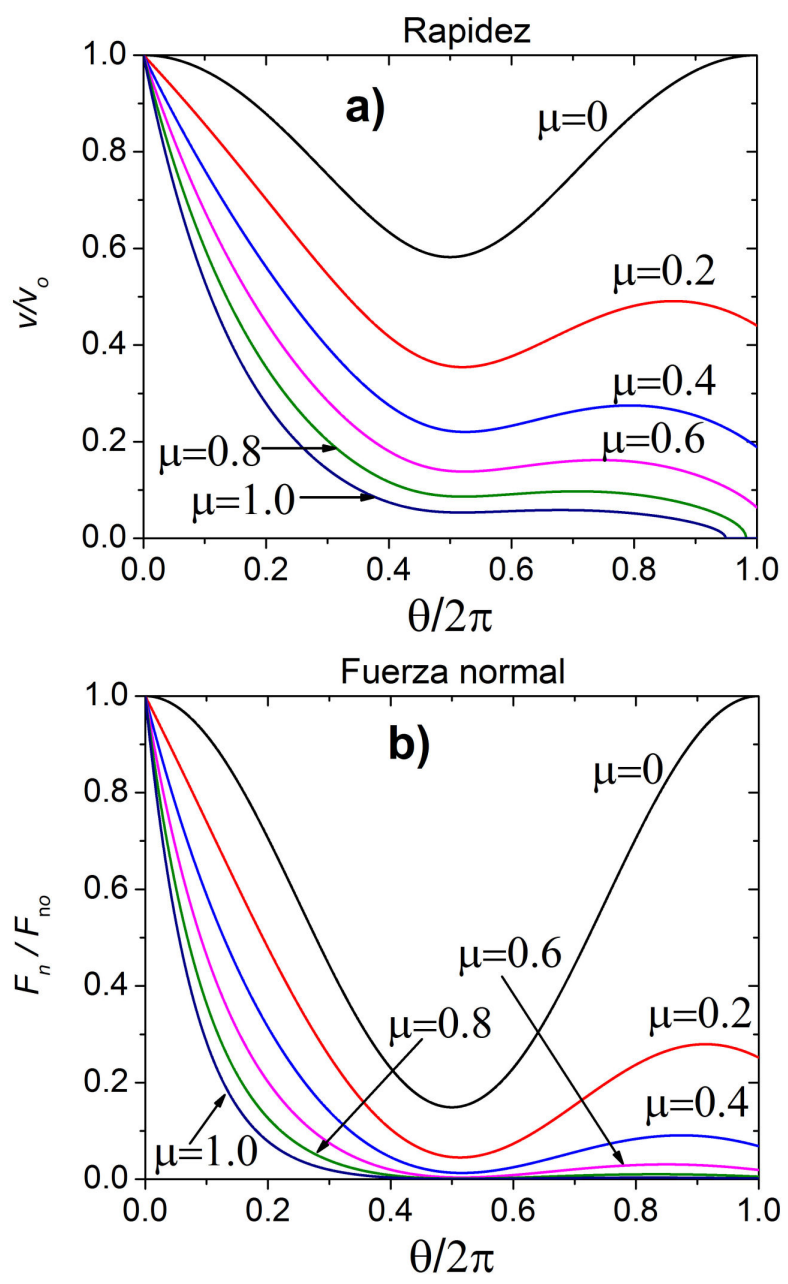

FIGURA 7. Comportamiento de la rapidez para diferentes valores del coeficiente de fricción cinética.

nuevamente a esta para $(\theta / 2 \pi)>0.65$, además, en este intervalo la amplitud del máximo presente en la segunda vuelta ha disminuido notablemente y el valor final de la rapidez decae hast el $6 \%$ del valor inicial. Finalmente, para $\mu \geq 0.8$ y $(\theta / 2 \pi)>0.35$ la fuerza normal es cero y la esfera pierde contacto con la trayectoria, sin embargo, continúa moviéndose en semicírculo con una rapidez constante hasta $(\theta / 2 \pi)>0.95$, en donde cae hasta tocar el suelo.

\section{Conclusiones}

En este trabajo se ha presentado el análisis teóricoexperimental sobre la dinámica del movimiento de una esfera que se mueve en una trayectoria circular vertical con fricción. En el estudio teórico, a partir de la segunda ley de Newton, se estableció una ecuación diferencial de Bernoulli para obtener la rapidez de la esfera a lo largo de la trayectoria circular. En el estudio experimental, se obtuvieron la rapidez y fuerza normal de la esfera de forma indirecta, a partir de mediciones de la posición de la esfera como función del tiempo. Los resultados experimentales muestran que la fricción produce una 
dismunición del $40 \%$ y $60 \%$ del valor inicial para la rapidez y fuerza normal, respectivamente. El ajuste de $\mu=0.12$ y $v_{0}=1.1 v_{P}$ permite describir de forma cualitativa los resultados experimentales obtenidos de forma indirecta para la rapidez y fuerza normal.

Finalmente, es importante mencionar que, el modelado matemático y la configuración experimental presentados, pueden utilizarse como herramienta didáctica en cursos de Física y ecuaciones diferenciales del primer y segundo ciclo universitario.

\section{Agradecimientos}

Los autores agradecen a las autoridades del TecNM / I.T. Apizaco por las facilidades brindadas para la elaboración de este proyecto. $i$. La forma general de una ecuación diferencial de Bernoulli es $\frac{d y}{d x}+p(x) y=f(x) y^{n}$.

1. Engineering Physics, (Krishna Prakashan Media), 2001. https://books.google.com.mx/books?id= 6MH7_FkIgD 4C

2. A. Bettini, A Course in Classical Physics 1-Mechanics, Undergraduate Lecture Notes in Physics, (Springer International Publishing, 2016). https://books.google.com. $\mathrm{mx} /$ books? id=6TTeCwAAQBAJ

3. F. d. Simoni, Velocidade mínima para completar um loop circular vertical: uma abordagem cinemática, Rev. Bras. Ensino Fís. 40 (2018). http://www.scielo. br/scielo.php?script=sci_arttext\&pid= S1806-11172018000300403\&nrm=iso https:// doi.org/10.1590/1806-9126-rbef-2017-0330

4. I. A. C. Melnik y V. d. A. Oliveira, Análise do movimento de uma partícula em um loop circular vertical usando nooes de limite, Rev. Bras. Ensino Fís. 42 (2020). http://www.scielo.br/ scielo.php?script=sci_arttext \&pid= S1806-11172020000100439\&nrm=iso https:// doi.org/10.1590/1806-9126-RBEF-2019-0244

5. L. P. Franklin y P. I. Kimmel, Dynamics of circular motion with friction, Am. J. Phys. 48 (1980). https://doi.org/10. $1119 / 1.12306$
6. I. Richard Lapidus, Circular motion with sliding friction, Am. J. Phys. 49 (883) (1981). https://doi.org/10.1119/1. 12388

7. P. L. T. Jr., Trouble on the loop-the-loop, Am. J. Phys. 55 (1987).https://doi.org/10.1119/1.14997

8. T. Prior y E. J. Mele, A block slipping on a sphere with friction: Exact and perturbative solutions, Am. J. Phys. 75 (2007). https://doi.org/10.1119/1.2410018

9. B. B. Asavapibhop y N. Suwonjandee, Loop-the-loop: An easy experiment, a challenging explanation, AIP Conference Proceedings 1263 (2010). https://doi.org/10.1063/1. 3479881

10. N. Suwonjandee y B. Asavapibhop, Loop-the-loop: bringing theory into practice, Phys. Educ. 47 (2012). https : //doi. org/10.1088/0031-9120/47/6/751

11. W. K lobus, Motion on a vertical loop with friction, Am. J. Phys. 79 (2011). https: / /doi.org/10.1119/1.3602091

12. D. Souza y V. Coluci, The motion of a ball moving down a circular path, Am. J. Phys. 85 (2017). https : / / doi .org/10. $1119 / 1.4972177$

13. O. Bertran y J. Riba, A revised solution for a sphere rolling in a vertical loop, Eur. J. Phys. 42 (2020). https: / / doi.org/ $10.1088 / 1361-6404 / a b b c a 1$ 